\title{
Arc Behavior and Droplet Dynamics of AC GTAW-GMAW Hybrid Indirect Arc
}

\author{
The effects of the AC waveform parameters, and the position of the wire and \\ tungsten electrodes on the arc behavior and droplet dynamics, were studied
}

\author{
S. J. CHEN, L. W. WANG, J. XIAO, AND P. S. WEI
}

\begin{abstract}
The alternating current (AC) gas tungsten arc welding-gas metal arc welding (GTAW-GMAW) hybrid indirect arc process is characterized by the arc configuration in which the AC flows between the filling wire and tungsten electrode but not the base metal. In this study, the effects of the square AC waveform parameters and the relative position of the wire and tungsten electrodes on the arc behavior and droplet dynamics were studied. Observations showed that the arc configuration between the tungsten electrode and wire on the wire-negative stage was in a curved cylinder shape, accompanied by cathode spot climbing along the droplet and wire surface, and the droplet swings toward the tungsten side. When the polarity of the wire was changed from cathode to anode, the arc configuration changed into a triangle profile. The droplet began to swing toward the wire side. The transport direction of the droplet was along the line connecting the tungsten electrode tip and wire tip. The results showed the effects of current frequency on the maximum climbing height of cathode spot. The cathode spot movement was also scaled, which is related to spread of current into the droplet.
\end{abstract}

\section{KEYWORDS}

- GTAW-GMAW Hybrid Indirect Arc • Cathode Spot • Droplet Dynamic

- Arc Behavior - Cathode Spot Motion

\section{Introduction}

Gas tungsten arc welding (GTAW) and gas metal arc welding (GMAW) are the most popular gas-shielded arc welding techniques used in many industrial fields. Traditional GTAW is a high-quality process that features less spatters and better weld bead surfaces than GMAW. However, GTAW suffers from slow speed and small amounts of deposited metal. Different methods, such as hot-wire GTAW (Ref. 1), activated-GTAW (Ref. 2), double-sided GTAW (Ref. 3), and twin-tungsten GTAW (Ref. 4), have been developed to obtain better deposition rates. On the other hand, GMAW is a process with high rates of metal deposition, but increased heat input into the base metal (Ref. 5). As a consequence, the GTAWGMAW hybrid welding process has become a new type of arc welding process combining the high quality of GTAW with the high efficiency of GMAW.

Kanemaru et al. (Refs. 6, 7) experimentally and numerically studied the balance of current between gas tungsten and gas metal arcs in GTAWGMAW hybrid welding. A suitable range of currents was confirmed. Chen et al. (Ref. 8) analyzed distributions of heat flux and weld formation, and proposed energy-force models to study interacting forces between gas tungsten and gas metal arcs in GTAW-GMAW hybrid welding. Tao et al. (Ref. 9) further studied the properties of hybrid arc, transition modes of droplets, and influence of different polarity connected to the weld joint. The GTAWGMAW hybrid arc could be superimposed in parallel gas tungsten and gas metal arcs. Similar to traditional arc welding, welding current is still from a consumable electrode or tungsten electrode to base metal through which heat input cannot be reduced effectively. Y. M. Zhang proposed a doubleelectrode-GMAW and arcing wire GTAW (Refs. 10, 11) in which a third consumable or nonconsumable electrode was added, and thus a bypass arc was introduced burning between it and the original wire or tungsten electrode. As a result, the total current flowing through the wire and the base metal could be separately controlled; in other words, the wire deposition rate and the heat input to the base metal were separately controlled or deeply decoupled.

Wang et al. (Ref. 12) studied the consumable-aided tungsten indirect arc welding with direct current (DC). An indirect arc was generated between the consumable electrode of the GMAW gun and tungsten electrode of the GTAW torch, whereas it was absent between the tungsten or consumable electrode of welding torch and base metal. Welding current flowed from the consumable electrode to tungsten electrode in the freeburning indirect arc. Consumable-aided tungsten indirect arc welding not only rapidly melted the welding wire but also 


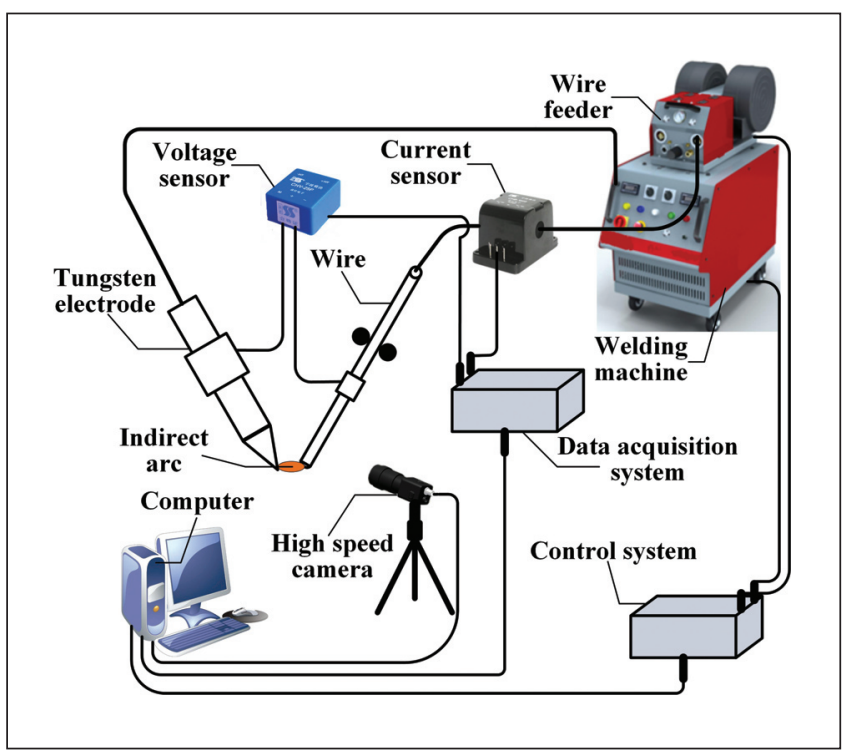

Fig. 1- Experimental setup.

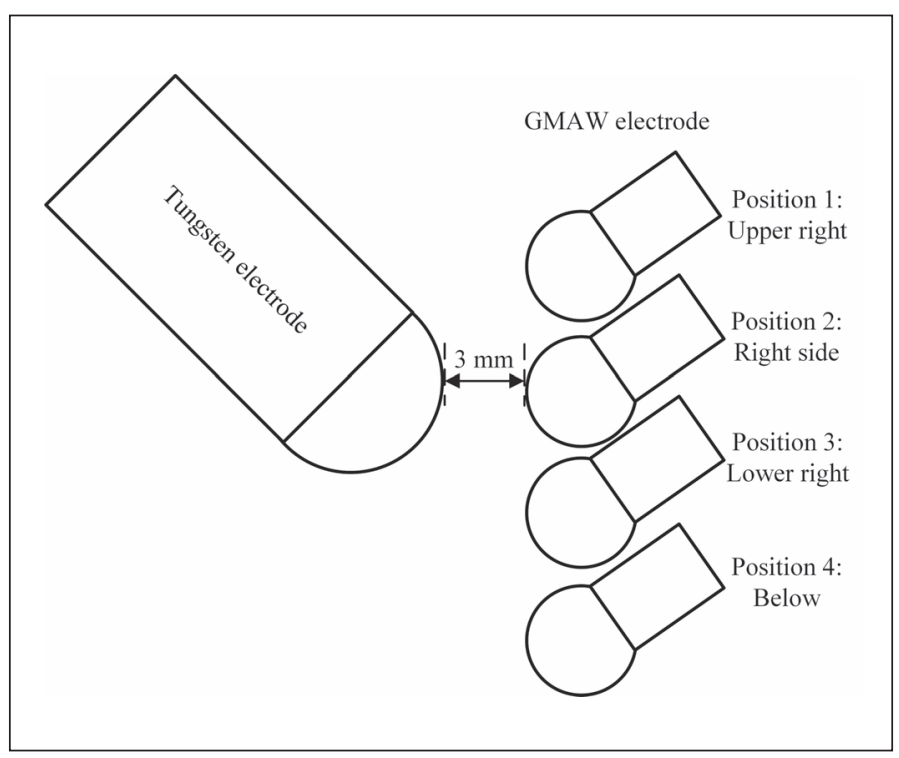

Fig. 2 - Initial relative positions of tungsten and wire. effectively restrained heat input into the base metal. The consumable electrode connected to the positive pole of welding power is the arc anode. It was found that the consumable electrode acting as the cathode had a higher melting rate than the anode (Refs. 13, 14). By comparison to tungsten-consumable indirect arc welding (TCIAW) with DC, AC TCIAW has a higher deposition rate, as also verified by theoretical analysis (Ref. 14).

In contrast to base metal as an arc terminal in conventional arc welding, arc terminals of tungsten and wire tip in TCIAW are comparatively small in size and allowed flexible variations. The alternative polarity between tungsten and wire tips in AC TCIAW also plays an important role in sustaining the arc between the two tips. Arc behavior and droplet transfer have an important effect on heat input, deposition rates, and weld forming. Understanding the different parameters affecting arc behavior and droplet transfer of AC TCIAW is essentially required for a practical use of the promising welding technology.

Defects such as porosity, weld cracking, or entrainment of an oxide layer into the weld pool occur if the oxide layer remains on a weld pool surface in arc welding. The oxide film on the surface of the base material can be removed through the cleaning action of the cathode spot, when the electrode is positive and the base material acts as a cathode (Ref. 15). Whether the motion is random or directed depends on a self-induced and external imposed magnetic field (Ref. 16). The behavior or motion of the cathode spot is thus directly linked to current distribution in arc plasma and strongly affects the detachment of the droplet on the wire tip.

In this paper, experiments were conducted on the effects of magnitude and frequency of welding current, relative location of the wire, and the tungsten tip on arc behavior and droplet transfer. Understanding and controlling arc behavior, cathode spot, and droplet transport are required for different kinds of arc welding processes.

\section{Experiment Setup}

The measurement system is composed of the following: welding power source, wire feeder, high-speed camera, current and voltage sensors, data acquisition, and control systems, as sketched in Fig. 1. The tungsten and wire, respectively, were connected with the two electrodes of the welding power supply. The arc was burning between the tungsten and wire with $\mathrm{AC}$. No current flowed to the base metal. The AC power source and wire feeder were both program-controlled. The argon shielding gas had a flow rate of 15 L/min. An IDT Motion Pro Y-4 highspeed camera was used to capture the arc and droplet dynamics. The ER70S6 wire had a diameter of $1.2 \mathrm{~mm}$, whereas the tungsten electrode had a diameter of $5.0 \mathrm{~mm}$. The current amplitudes of the AC square wave were $80,100,120,140$, and $160 \mathrm{~A}$. The AC frequencies varied in four levels: 25 , 50, 100, 200, and $400 \mathrm{~Hz}$.

The initial relative positions between the tungsten and wire tip before arc ignition were categorized to four configurations: wire at upper right, right side, lower right, and below, as shown in Fig. 2. The initial distance between two tips was $3 \mathrm{~mm}$ in the horizontal direction. With a given welding current, the arc between the tungsten and wire electrode was ignited by way of short circuit.

\section{Results and Discussion}

The recorded waveforms of current and voltage showed good periodicity and repeatability, which verified the expected stability of indirect arc and current waveform.

\section{Cathode Spot Motion}

In Tests 1-5, the welding current was fixed at $100 \mathrm{~A}$. Current alternating frequency of the square wave increased from 25, 50, 100, and 200 to $400 \mathrm{~Hz}$. The time ratio of positive and negative half-wave was 1:1 (tungstenpositive/wire-negative stage is defined as positive-half-wave). The remaining test parameters are shown in Table 1.

Figure 3 shows time-dependent current and voltage with positive and 


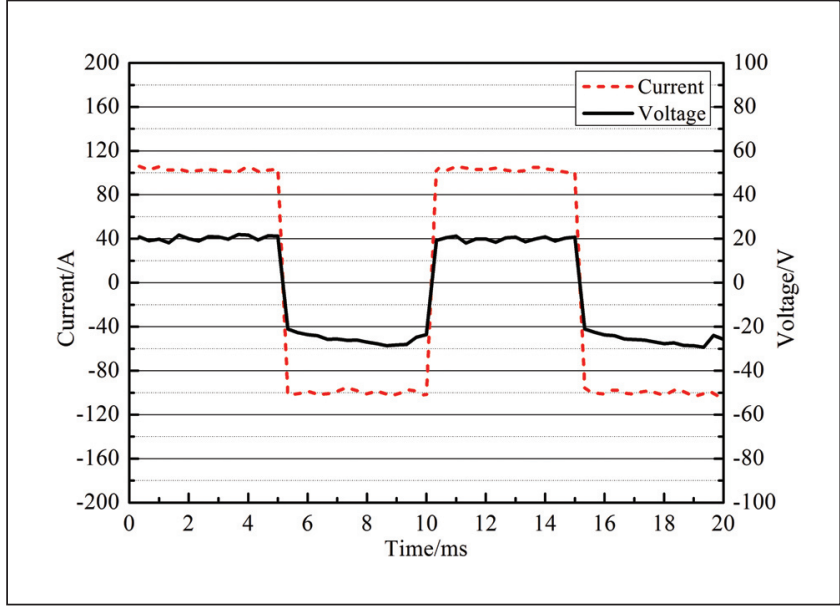

Fig. 3 - Recorded current and voltage waveforms in Test 3.

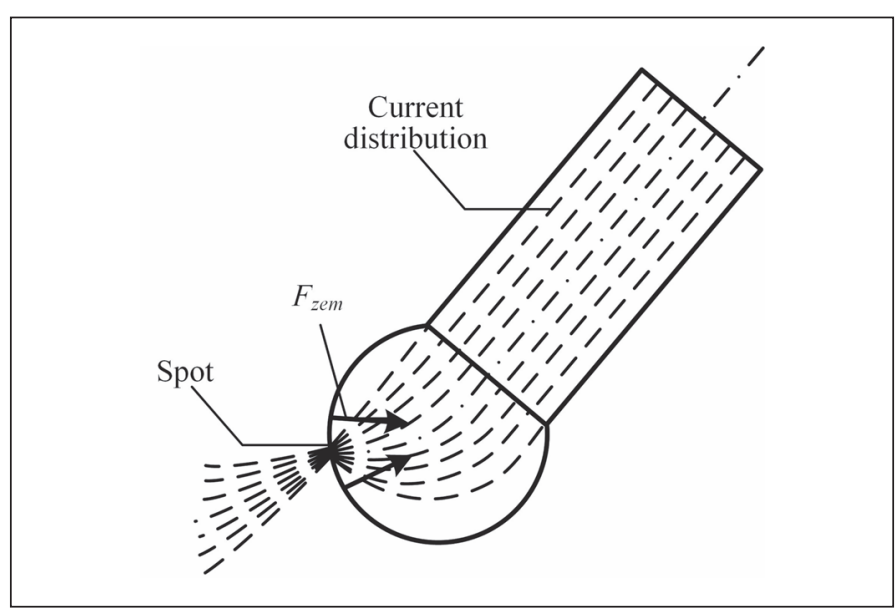

Fig. 4 - Schematic of current distribution and electromagnetic force at the spot. negative half-waves during the indirect arc burning process (see Test 3 ). It can be seen that duration of positive and negative half-wave is $5 \mathrm{~ms}$ in each cycle, respectively, corresponding to a square current frequency of $100 \mathrm{~Hz}$. The current was stable at about $100 \mathrm{~A}$. The voltage of positive half-wave was maintained between 18 and $22 \mathrm{~V}$, whereas the voltage of negative halfwave showed a gradual increase trend from 21 to 29 V. A sudden decline occurred near the end of each cycle because when the wire is cathode, the cathode spot automatically searches for the oxide film and climbs along the wire. In view of the cathode spot climbing, the voltage of the indirect arc rose as the arc length increased. The upward motion of the cathode spot has been attributed to the pressure difference across the cathode spot (Ref. 16). Pressure difference across the cathode spot, considering the same order of magnitude as electromagnetic force, was found to be proportional to the products of external magnetic field and self-magnetic field. Electric current was then scaled by a self-magnetic field and external magnetic field, satisfied by Ampere's law and Lorentz's force law. The resultant force thus resulted in an upward motion of the cathode spot.

However, in this study, it is difficult to exclusively specify the external magnetic field due to the complex arc dynamics, thereby, an alternative and simpler interpretation by using scale analysis was proposed. Fluid flow equation in the arc beyond the droplet was given by (Ref. 16)

$\rho_{a} V_{r^{\prime}} \frac{d V_{r^{\prime}}}{d r^{\prime}}=-j_{n^{\prime}} B_{\theta^{\prime}}$

where variables $\rho_{a}, V_{r^{\prime}}, j_{n^{\prime}}$, and $B_{\theta^{\prime}}$ are density, radial velocity, axial current density, and azimuthal self-induced magnetic field in arc, respectively.

Equation 1 indicates that the pinch effect takes place in the arc ahead of the droplet, as a result of negative velocity in the radial direction. Current spreads after entering the cathode spot, as illustrated in Fig. 4. Fluid equation in the droplet yield $\rho_{d} V_{s} \frac{d V_{s}}{d s}=j_{r} B_{\theta}$

where $\rho_{d}$ is density of the droplet, subscripts $s$ and $r$ are, respectively, the direction parallel to the droplet surface and radial direction in the droplet.

Since current is spread in the droplet, Equation 2 indicates that liquid velocity in the droplet increases for positive current density in the radial direction. Ampere's law related to Equation 2 is

$$
-\frac{\partial B_{\theta}}{\partial s}=\mu_{d} j_{r}
$$

Scaling Equations 2 and 3 gives

$$
V_{s} \sim B_{\theta} \sim j_{r} \sim I
$$

where $I$ is the welding current.

Equation 4, therefore, shows the cathode spot moved upward as a result of a spread of electric current in the droplet. Spot velocity increased linearly with magnetic field agreed with pre-

Table 1 - Test Parameters for Different Current Frequencies

Test No.

Current

Amplitude (A)
Current

Frequency $(\mathrm{Hz})$
Wire Feed

Speed

(m/min)
Relative Location Between Tungsten and Wire Tip

\begin{tabular}{lllll}
\hline 1 & 100 & 25 & 3.2 & Right side \\
2 & 100 & 50 & 3.2 & Right side \\
3 & 100 & 100 & 3.2 & Right side \\
4 & 100 & 200 & 3.2 & Right side \\
5 & 100 & 400 & 3.2 & Right side \\
\hline
\end{tabular}



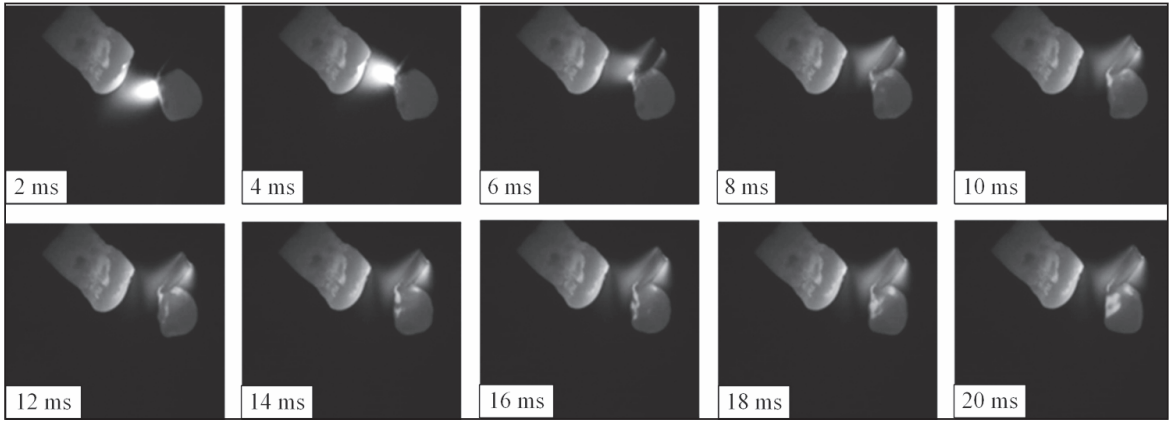

Fig. 5-A scenario of cathode spot climbing in search of oxide film.
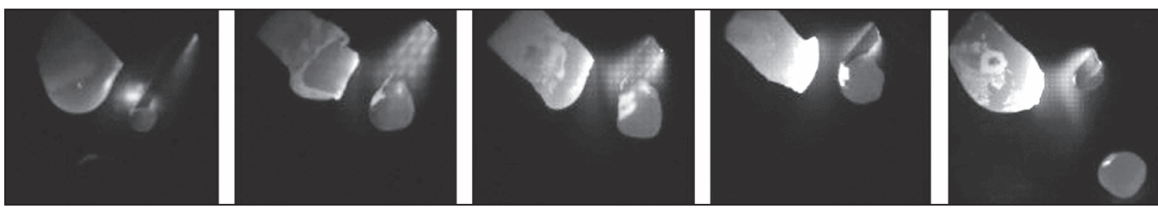

Fig. 6-Cathode spot climbing at different AC frequencies.

vious measurements (Refs. 17-19) and arc current (Ref. 19).

\section{The Effects of Current Frequency on Arc Behavior and Droplet Dynamics}

Observation from photos in Fig. 5 shows that when polarity of the wire changed from anode to cathode, cathode spots moved upward along the droplet surface and automatically searched for oxide films. Figure 6 shows the climbing behavior of cathode spots at different frequencies of the $\mathrm{AC}$ square wave (see Tests $1-5$ ). Since the AC frequency directly determined the wire-negative duration that actually determines the available time for cathode spot climbing in each AC cycle, the following would discuss the influence of $\mathrm{AC}$ frequency, rather than the wirenegative time, on cathode spot climbing for ease of understanding since the frequency is generally the first-mind parameter for depicting an $\mathrm{AC}$ waveform.

It can be seen that as current fre- quency gradually increased, the cycle of the square wave and wire-negative duration decreased, and the climbing height of the cathode spot decreased. The maximum climbing height along the wire direction, defined as the distance between the wire tip and arc root position on the wire, can be obtained by calibrating the measured pixels to $\mathrm{mm}$. Figure 7 shows that the maximum climbing height of the cathode spot was $8.1 \mathrm{~mm}$ at $25 \mathrm{~Hz}$ $\mathrm{AC}$ waveform (20 ms wire-negative time in each cycle, see Test 1 ) and decreased to $1.7 \mathrm{~mm}$ at $400 \mathrm{~Hz} \mathrm{AC}$ waveform (1.25 ms wire-negative time in each cycle, see Test 5 ). The maximum climb height, therefore, increased as AC frequency decreased because the cathode spot had more time to search oxide films when the $\mathrm{AC}$ frequency was lower.

The trend of the maximum arc voltage during the wire-negative half-wave is similar to that of the maximum climbing height of the cathode spot, as shown in Fig. 8, because the arc length increased as the cathode spot climbed. In this sense, being accompanied with the cathode spot climbing, the arc voltage was increased too but not keeping relatively stable, which has already been demonstrated by Fig. 3 . Therefore, the arc voltage increasing amplitude and thus the maximum arc voltage during the wire-negative half wave would be affected by the AC frequency because the wire-negative duration is directly determined by the $\mathrm{AC}$ frequency. Lower current frequency means more time for the cathode spot to climb and arc length to increase. At $400 \mathrm{~Hz}$ current (1.25 ms wire-negative time, see Test 5), the maximum arc voltage during the wire-negative half wave was $32 \mathrm{~V}$, and the voltage increase amplitude during climbing was $3.5 \mathrm{~V}$. As the AC frequency was reduced to $25 \mathrm{~Hz}$ (20 ms wire-negative time, see Test 1 ), the maximum arc voltage increased to $40 \mathrm{~V}$, and voltage increase amplitude increased to $14 \mathrm{~V}$.

\section{The Effects of Current Magnitude on Arc Behavior and Droplet Dynamics}

To study the effects of current magnitude on arc behavior and oscillation of droplets, test parameters denoted by Tests 6-10 are shown in Table 2. The current frequency was fixed at $100 \mathrm{~Hz}$, whereas the current magnitudes were $80,100,120,140$, and $160 \mathrm{~A}$, respectively. Figures 9-13 show the corresponding shape and brightness of the tungsten-consumable indirect arc at different welding currents. The tungstenconsumable indirect arc was found to be in a "V" shape. The brightness and size of the indirect arc gradually increased as welding current increased.

Figures 9-13 show oscillations of droplets under the action of arc repulsive force for different magnitudes of current in a square-wave cycle in Tests $6-10$. A common phenomenon was re-

Table 2 - Test Parameters at Different Current Magnitudes

\begin{tabular}{ccccc} 
Test No. & $\begin{array}{c}\text { Current } \\
\text { Magnitude (A) }\end{array}$ & $\begin{array}{c}\text { Current } \\
\text { Frequency (Hz) }\end{array}$ & $\begin{array}{c}\text { Wire Feed } \\
\text { Speed } \\
\text { (m/min) }\end{array}$ & $\begin{array}{c}\text { Relative Position } \\
\text { Between Tungsten } \\
\text { and Wire Tip }\end{array}$ \\
\hline 6 & 80 & 100 & 2.6 & Right side \\
7 & 100 & 100 & 3.2 & Right side \\
8 & 120 & 100 & 4.4 & Right side \\
9 & 140 & 100 & 5.6 & Right side \\
10 & 160 & 100 & 7.0 & Right side \\
\hline
\end{tabular}




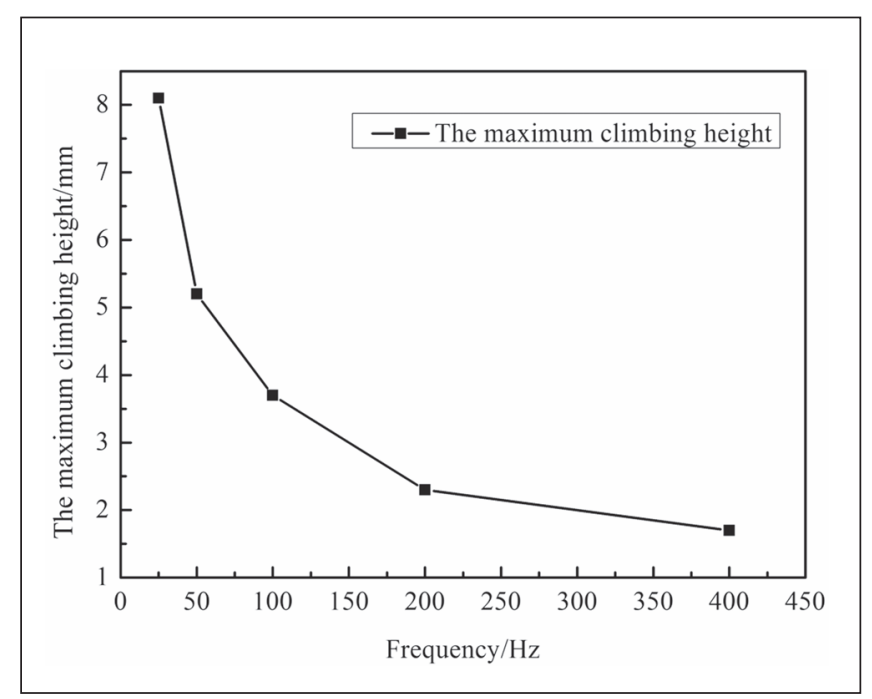

Fig. 7- Effect of AC frequency on maximum cathode spot climbing height.
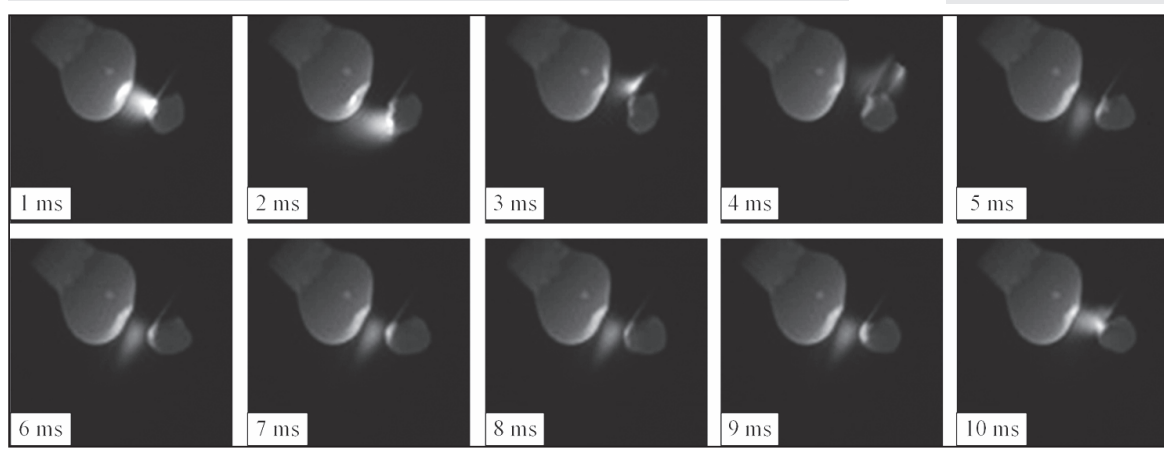

Fig. 9-Oscillation of a droplet within a cycle at $80 \mathrm{~A}$.
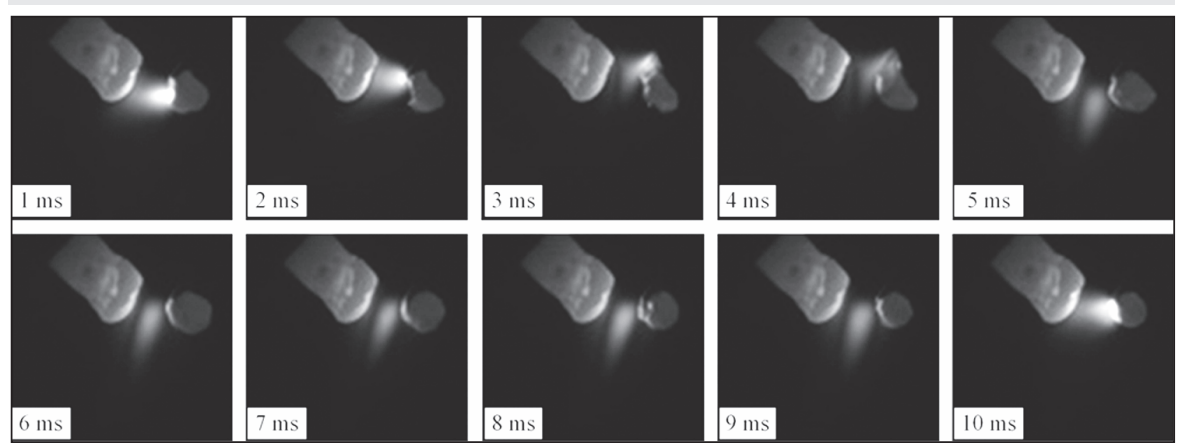

Fig. 10 - Oscillation of a droplet within a cycle at $100 \mathrm{~A}$.

vealed by examining five groups of photos. The first four pictures in each group were at the wire stage in cathode polarity. The first picture at $1 \mathrm{~ms}$ was the moment of wire changing polarity from anode to cathode, whereas the second to fourth pictures (from 2 to $4 \mathrm{~ms}$ ) were on the climbing stage of the cathode spot. As the cathode spot climbed, the spot force responsible for the deviation of the droplet toward the tungsten electrode decreased (see re- duction of arc brightness), and resulted in swing toward the tungsten side. The fifth picture at $5 \mathrm{~ms}$ was the moment the wire changed polarity from cathode to anode. It can be seen that arc configuration was changed from a cylinder shape into a triangular profile - Fig. 13. The sixth to ninth pictures (from 6 to $9 \mathrm{~ms}$ ) show a stable anode spot maintained on the upper part of the droplet. Under the action of the anode spot force, the droplet swung toward the wire side until polarity of the wire was changed from anode to cathode at $10 \mathrm{~ms}$ (the tenth picture). A sudden change in polarity from anode to cathode enhanced arc brightness and changed arc configuration from a triangle shape into a curved cylinder shape, and increased deflection of the droplet toward the wire side. The droplet completed the oscillation process in a square wave current cycle.

In the case of a current magnitude of $80 \mathrm{~A}$ and the wire as a cathode, the droplet is biased toward the right side of the wire under the action of the cathode spot force. After polarity of the wire was changed to the anode under the action of the anode spot force, the droplet could swing to just below the end of the wire, indicating that the anode spot force acting on the droplet at the same welding current was less than the cathode spot force. In the case of a current magnitude of $100 \mathrm{~A}$, the droplet was always on the right side of the wire end and could not swing to just below the end of the wire, regardless of polarity of the wire during one cycle. When current magnitude was higher than $100 \mathrm{~A}$, and the wire was on the stage of anode polarity, the droplet could not swing to just below the end of the wire. Deflection of the droplet toward the right side of the wire increased with the welding current, indicating that the spot force increased with the welding current.

Figure 14 shows the period and diameters of detached droplets for dif- 

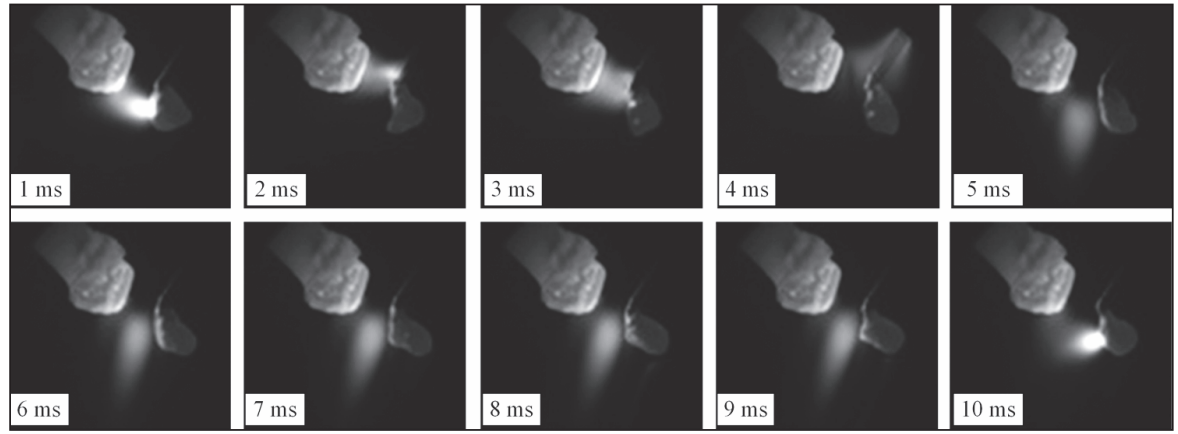

Fig. 11 - Oscillation of a droplet within a cycle at $120 \mathrm{~A}$.
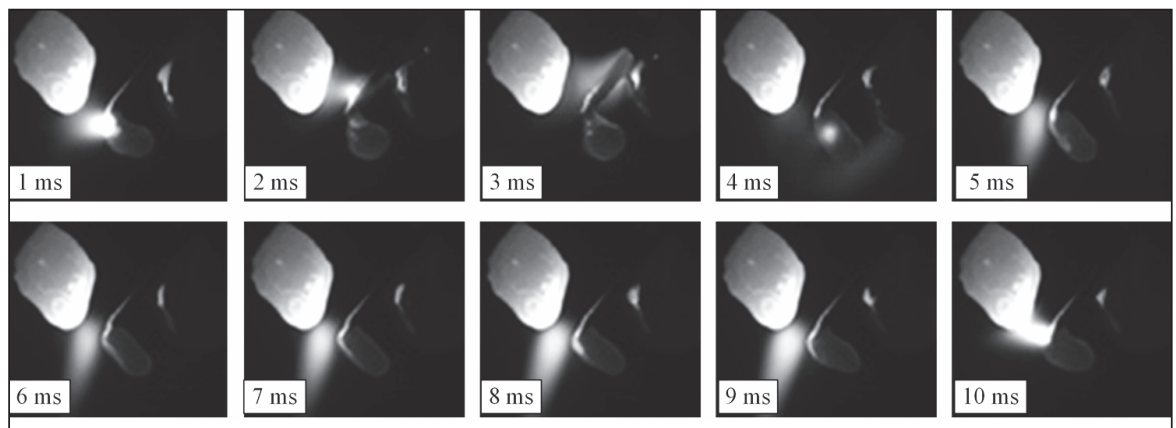

Fig. 12 - Oscillation of a droplet within a cycle at 140 A.
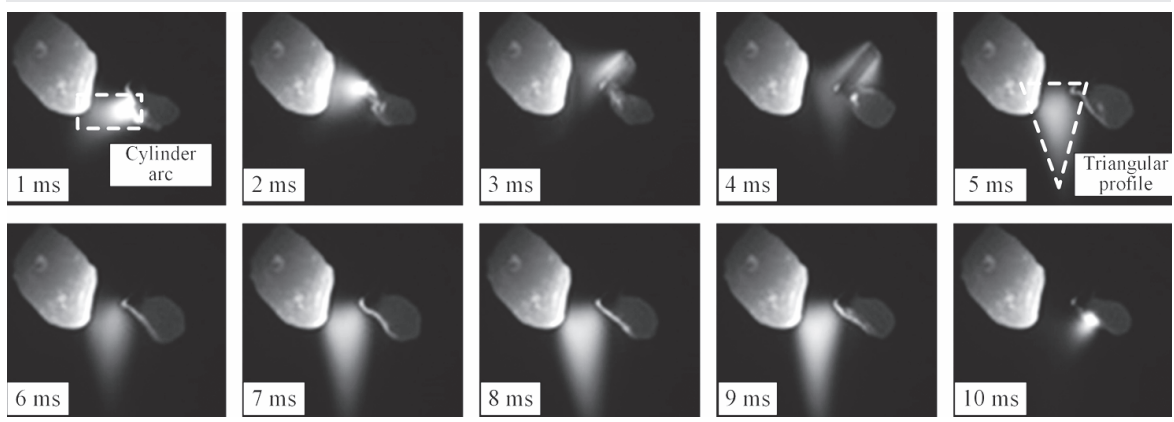

Fig. 13 - Oscillation of a droplet within a cycle at $160 \mathrm{~A}$.

ferent currents. It can be seen that an increase in current reduced the period between the droplet detaching and diameter of the detached droplet.

There are different types of detaching or retarding forces responsible for the dynamic behavior of the droplet in the tungsten-consumable indirect arc. Forces acting on the droplet include gravitational force $F_{g}$, surface tension $F_{\sigma}$, electromagnetic force $F_{e m}$, spot force $F_{v}$, and aerodynamic drag force $F_{d}$, as shown in Fig. 15.

1. Gravitational force, $F_{g}$

The force due to gravity can be expressed as

$F_{g}=\rho_{d} g V_{d}$

where $V_{d}$ and $g$ are, respectively, droplet volume and gravitational acceleration. Gravitational force during welding always promotes detachment of droplets.

2. Surface tension, $F_{\sigma}$

Surface tension is given by (Ref. 20)

$$
F_{\sigma}=2 \pi r_{w} \gamma e_{w}
$$

where $r_{w}$ is the radius of the wire, $\gamma$ is the surface tension coefficient, and $e_{w}$ is the unit vector in the wire direction. Surface tension is the force to keep the droplet at the tip of the wire, impeding detachment of the droplet.

\section{The spot force, $F_{v}$}

The indirect arc results in the polar spot at the cathode or anode spots on the droplet. Pressure caused by the impact force of electron and ion flow, and recoil force of metal vapor on the spot, is called the spot force. Since spots occur on the left side of the droplet, the impact force of electron and ion flows, and recoil force of metal vapor, pushed the droplet toward the right side (see Fig. 13), and broke the droplet from the wire. In view of the deviation from the transition direction, spot force, $F_{v}$, impedes droplet transfer.

4. Electromagnetic force, $F_{e m}$

Electromagnetic force or Lorentz force can be calculated from

$F_{e m}=\int j \times B d V_{d}$

Consider uniform current density $j$ in $k$ direction on any cross section. Ampere's law $\left(\partial B_{\theta} / \partial r\right) / r=\mu j$ gives azimuthal magnetic field induction $B_{\theta}=$ $\mu j r / 2$. Equation 7 therefore gives

$$
\begin{aligned}
& F_{e m}=\int j k \times B_{\theta} e_{\theta} d V_{d}=-e_{r} \int j B_{\theta} d r d A_{r} \\
& =-e_{r} \frac{1}{4} \mu j^{2} A_{r}\left(r_{\text {max }}^{2}-r^{2}\right)
\end{aligned}
$$

where magnetic force at the surface vanishes because of zero current density. Radius $r_{\max }$ is the maximum radius of cylindrical arc. Equation 8 indicates force due to pinch effect. Electromagnetic force from Equation 7 can be projected into any direction, for example, radial and horizontal directions, $F_{r e m}$ and $F_{z e m}$, by simply taking the inner product with unit vectors of radial and horizontal directions. Radial component of electromagnetic force, $F_{\text {rem }}$, necks the droplet, promoting separation of the droplet from the wire. In view of pinch force, electromagnetic force from the small to large section results in the component of magnetic force, $F_{z e m}$, toward the right direction (see Fig. 15), leading to droplet breaking away from the wire.

5. Aerodynamic drag force $F_{\text {drag }}$

Aerodynamic drag force can be expressed as (Ref. 20)

$$
F_{\text {drag }}=\frac{1}{2} \pi v_{a}^{2} \rho_{a} r_{d}^{2} C_{d r a g} \frac{v_{a}}{\left\|v_{a}\right\|}
$$

where the aerodynamic drag coefficient, $C_{\text {drag }}$, related to Reynolds number, $R e$, is given by

$$
C_{\text {drag }}=\frac{24}{R e}+\frac{6}{\sqrt{1+R e}}+0.4
$$

The higher the droplet radius and plas- 


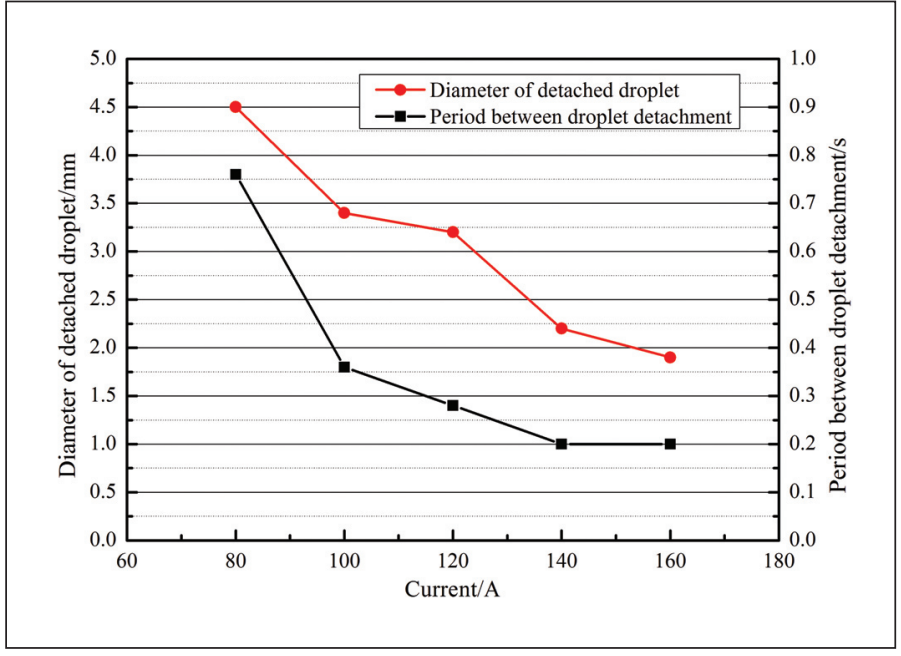

Fig. 14 - Periods and diameters between droplet detachments at different currents.

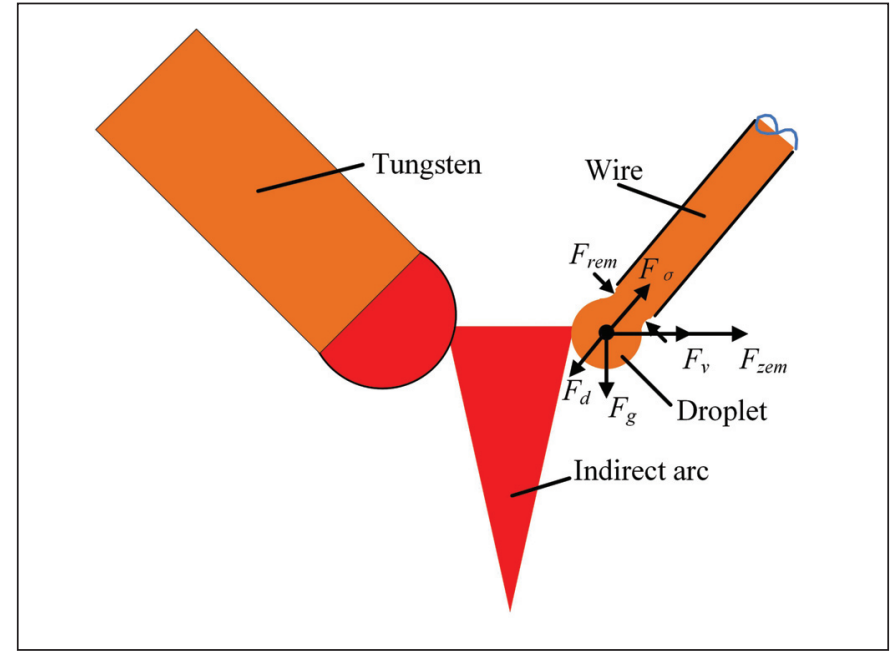

Fig. 15 - Forces acting on a droplet in the tungstenconsumable indirect arc.
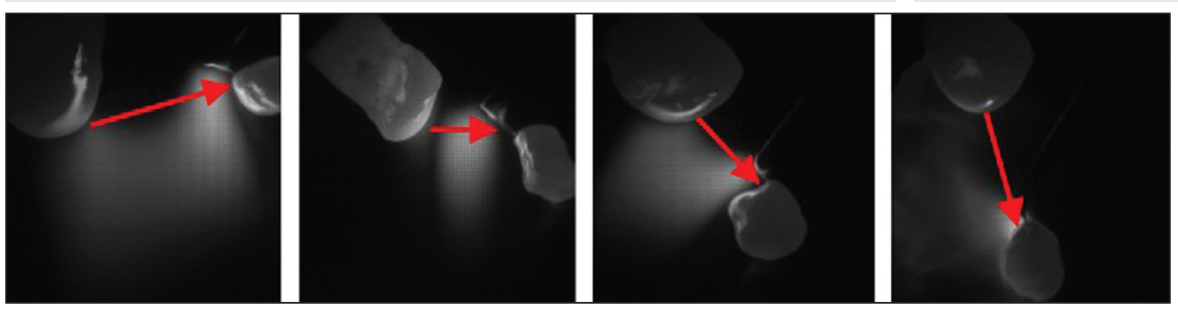

Fig. 16 - The direction of droplet detachment at different relative positions between the tungsten and wire tips: $A$ - Upper right; B - right side; $C$ - lower right; $D$ - below.

ma velocity, the higher the aerodynamic force is. In view of the plasma flow from the end of the wire to the droplet, the aerodynamic drag force is the force to promote droplet transfer.

\section{The Effects of Relative Location between Tungsten and the Wire Tip on Droplet Transfer}

Whether with tungsten-consumable or twin-wire indirect arc (Ref. 13), under the acting of spot force, the droplet was repelled to both sides, rather than the force of the conven- tional arc welding, either in the wire feeding direction or opposite to the feeding direction. Tests 11-14 qualitatively analyzes the direction of spot force acting on the droplet by controlling the wire feed speed to change the relative position between the tungsten and wire tip, with a fixed current magnitude of $100 \mathrm{~A}$ and an AC frequency of $100 \mathrm{~Hz}$. The remaining test parameters are shown in Table 3 . It can be seen that an increase in wire feed speed resulted in the droplet from the upper right, right side, lower right, and below the tip of the tungsten electrode.

Figure 16 shows the photos at the moment of the droplet breaking away from the wire for different relative positions between the tungsten and wire tip via controlling the wire feed speed. When the end of the wire was located on the upper right of the tungsten tip, the droplet was transferred to the upper right of the tungsten tip after breaking away from the wire, as shown in Fig. 16A. When the end of the wire was located on the right side, lower right, and below the tungsten tip, directions of the droplet transport were at the right side, lower right, and below the tungsten tip, as shown in Fig. $16 \mathrm{~B}-\mathrm{D}$, respectively. In summary, the direction of droplet detachment after breaking away from the wire was along the line connecting the tungsten electrode and wire tip.

\section{Conclusions}

The following conclusions were drawn:

1. Arc configuration between the tungsten electrode and wire on the stage of cathode was in a curved cylin-

Table 3 - The Effect of Wire Feed on Relative Position Between Tungsten and Wire Tip

Test No. C Current Magnitude (A)
Current Frequency $(\mathrm{Hz})$
Wire Feed

Speed

(m/min)
Relative Position Between Tungsten and Wire Tip

\begin{tabular}{ccccc}
\hline 11 & 100 & 100 & 3.0 & Upper right \\
12 & 100 & 100 & 3.2 & Right side \\
13 & 100 & 100 & 3.5 & Lower right \\
14 & 100 & 100 & 3.6 & Below \\
\hline
\end{tabular}


der shape. The cathode spot appeared and climbed along the droplet surface. The spot force responsible for deviation of the droplet toward the tungsten electrode decreased, resulting in the droplet to swing toward the tungsten side.

2. As polarity of the wire was changed from cathode into anode, arc configuration was suddenly changed from the cylinder shape into a triangular profile. A stable spot was maintained on the upper part of the droplet. The droplet began to swing toward the wire side until polarity of the wire was changed from the anode to cathode.

3. Motion of the cathode spot was related to the spread of current into the droplet.

4. The forces on the cathode spot were greater than those on the anode spot.

5. The maximum climbing height of cathode spot, maximum arc voltage, and voltage increase amplitude during the wire-negative half-wave decreased as the $\mathrm{AC}$ frequency increased because increased $\mathrm{AC}$ frequency means less wire-negative duration in each $\mathrm{AC}$ cycle.

6. The transport direction of the droplet after breaking away from the wire was along the line connecting the tips of tungsten electrode and wire.

\section{Acknowledgments}

This work is supported by the Beijing Natural Science Foundation (Grant No. 3162004) and National Natural Science Foundation of China (Grant No. 51505009 and 51475009). We also acknowledge the Program of Invited Distinguished Professorship supported by IDHT20150210, No. 456, 2014, Beijing Education Committee, P. R. China.

\section{References}

1. Sykes, I., and Digiacomo, J. C. 1995. Automatic hot wire GTA welding of pipe offers speed and increased deposition. Welding Journal 74(7): 53-s to 56-s.

2. Yang, C., Lin, S., and Liu, F. 2003. Research on the mechanism of penetration increase by flux in A-TIG welding. J. Mater. Process. Technol. 19: 225-227.

3. Cao, H. M., Wu, L., and Dong, H. G. 2001. Current density distribution in double-sided GTAW process. J. Mater. Process. Technol. 17(1): 187, 188.

4. Leng, X. S., Zhang, G. J., and Cao, H. M. 2006. A study on twin-tungsten TIG welding method. China Weld. 15(1): 49-52.

5. Kamiya, H., Fujita, T., and Enjo, Y. 1985. Kikkuchi: Oxygen content and fracture toughness on MIG weld metal of SUS 304 steel. Quart. J. Japan Weld. Soc. 3(3): 138-145.

6. Kanemaru, S., Sasaki, T., and Sato, T. 2013. Study for TIG-MIG hybrid welding process. Weld. World 58(1): 11-18.

7. Kanemaru, S., Sasaki, T., and Sato, T. 2015. Study for the mechanism of TIGMIG hybrid welding process. Weld World. 59(2): 261-268.

8. Chen, J. 2016. Influence of arcs interaction on TIG-MIG hybrid welding process. Chin. J. Mech. Eng-En. 52(6): 59-64.

9. Tao, Y., Zhang, S., and Gao, H. 2012. Analysis of mechanism for TIG-MIG hybrid arc properties. Trans. China Weld. Inst. 33(7): 25-28.

10. Zhang, Y. M., Jiang, M., and Lu, W. 2004. Double electrodes improve GMAW heat input control. Welding Journal 83(11): 39 -s to 41 s.

11. Chen, J. S., Lu, Y., Li, X. R., and Zhang, Y. M. 2012. Gas tungsten arc welding using an arcing wire. Welding Journal 91(10): 261-s to 269-s.

12. Wang, J., Wang, Y. X., and Feng, J. C. 2009. A study on consumable aided tungsten indirect arc welding. China Weld. 18(3): 27-31.

13. Chen, S. J., Wang, L. W., and Wei, P. S. 2016. Sustaining the inter-wire arc in twin-wire indirect arc welding. J. Manuf. Process. 21: 69-74.

14. Chen, S. J., Zhang, L., and Bai, L. L. The welding method of twin-wire indirect arc. China patent application no. CN201310456520.9, 2013.

15. Tashiro, S., Yuji, T., Fujimaru, A., Kinoshita, H., Yasui, K., Bouno, T., Methong, T., and Tanaka, M. 2015. Optical observation of cathode spot in AC tungsten intert gas (TIG) welding on aluminum plate using helium. Trans. JWRI 44: 1-4.

16. Garner, A. L. 2008. Cathode spot motion in an oblique magnetic field. Appl. Phys. Lett. 92: 011505.

17. Waszink, J. H., and Piena, M. J. 1986. Experimental investigation of drop detachment and drop velocity in GMAW. Welding Journal 65(11): 289-s to 298-s.

18. Sherman, J. C., Webster, R., Jenkins, J. E., and Holmes, R. 1975. Cathode spot motion in high-current vacuum arcs on copper electrodes. J. Phys. D: Appl. Phys. 8: 696-702.

19. Perskii, N. E., Sysun, V. I., and Khromoi, D. Y. 1989. Dynamics of vacuumdischarge cathode spots. High Temp. 27: 832-839.

20. Lancaster, J. F. The Physics of Welding. Oxford, England: Pergamon Press.

SHUJUN CHEN, LIWEI WANG, JUN XIAO (jun.xiao@bjut.edu.cn), and PENGSHENG WEl are with the Engineering Research Center of Advanced Manufacturing Technology for Automotive Components, Ministry of Education, Beijing University of Technology, Beijing, China. WANG is also with the School of Materials Science and Engineering, Hebei University of Science and Technology, Shijiazhuang, China. In addition, WEI is with the Department of Mechanical and Electro-Mechanical Engineering, National Sun Yat-Sen University, Kaohsiung, Taiwan. 